\title{
SWKB and proper quantization conditions for translationally shape invariant potentials
}

\author{
Kamal Mahdi ${ }^{1}, Y$. Kasri ${ }^{1,2}$, Y. Grandati ${ }^{2}$ and A. Bérard ${ }^{2}$ \\ ${ }^{1}$ Laboratoire de Physique Théorique, Faculté des Sciences Exactes, \\ Université de Bejaia, 06000 Bejaia, Algérie. \\ ${ }^{2}$ Equipe BioPhysStat, Laboratoire LCP-A2MC, ICPMB, IF CNRS N02843, \\ Université de Lorraine, 1 bvd D.F. Arago, 57078 Metz, France.
}

\begin{abstract}
Using a recently proposed classification for the primary translationally shape invariant potentials, we show that the exact quantization rule formulated by $\mathrm{Ma}$ and $\mathrm{Xu}$ is equivalent to the supersymmetric JWKB quantization condition. The energy levels for the two considered categories of shaped invariant potentials are also derived.
\end{abstract}

Recently, a new quantization rule in quantum mechanics which leads to the exact spectrum for some exactly solvable systems was proposed by Ma and $\mathrm{Xu}[1]-[2]$. This rule brings a correction term to the well known JWKB quantization condition. This quantum correction is given in the form of an integral, which contains the solution of Riccati's equation associated to the Schrödinger's equation for the ground state. Qiang and Dong [3] have simplified the formulation of Ma-Xu rule, calling it the proper quantization rule. Since its original formulation by Ma and Xu in 2005, several papers have been published on its possible applications to different potentials. This rule has been applied successfully to numerous solvable potentials in one and three dimensions, as well as separable noncentral potentials [4. In fact it has been proven that the Ma-Xu formula is necessary exact for every primary translationally shape invariant potential (PTSIP) [5]. As recently discovered, there exist also infinite towers of secondary TSIP for which the Ma-Xu quantization rule has not been proven to be exact [6, 7, 8,

Another quantization rule based on supersymmetric quantum mechanics, the so-called SWKB rule [9]-[11], permits the calculation of energy levels. It is also exact for every PTSIP but not for secondary TSIP [12].

Until now, the question about a direct link between the two quantization rules remains still unclear. The main purpose of this paper is to examine this link. Adopting the classification into two categories of PTSIP introduced in [13, we show that, for these potentials, the proper quantization rule is in fact equivalent to the SWKB condition. Starting from the SWKB condition, by means of some transformations and using appropriate changes of variables, we derive the proper quantization formula and then establish a direct and explicit connection between the two quantization rules in the class of potentials for which they appear to be exact. The quantization formulas allow then to obtain global formulas for the energy spectrum of the two categories of PTSIP and we apply it to retrieve the energy levels of the generic PTSIP.

The paper is organised as follows: the classification into two categories of potentials is introduced in the first section, then a brief introduction of the two quantization formulas is presented in the second section. In the next paragraph, the connection between these two rules is established. As an application, we calculate the energy levels for the two considered categories of potentials in the last section. 


\section{Potentials of first and second category}

Based on an analysis of the structure of PTSIP, Grandati and Berard [13] have shown that these potentials can be classified into two categories, simplifying the preceding classifications proposed initially by Gendenshtein [16] and later by Barclay [17]. In what follows, we use this classification. The basic idea is that every PTSIP can be reduced into a second degree polynomial or a second degree Laurent polynomial via a uniquely defined change of variable.

\section{$1.1 \quad$ First category (generic)}

We say that a one-dimensional potential $V(x)$ is a generic potential of the first category [13] if, with a suitable choice of a function $y(x)$, the two following conditions on the potential and $y$ are fulfilled

$$
\left\{\begin{array}{l}
V(x) \rightarrow V(y)=\lambda_{2} y^{2}+\lambda_{1} y+\lambda_{0}, \lambda_{2}>0 \\
y^{\prime}(x)=\alpha\left(1 \pm y^{2}\right)>0
\end{array}\right.
$$

In this category we find the following potentials : Rosen-Morse I, Rosen-Morse II and Eckart. It also contains the three exceptional cases corresponding to the harmonic, Morse and effective radial Kepler-Coulomb potentials.

We now seek the superpotential associated to this category. For a particle subjected to a potential $V(x)$ with a bound state spectrum $\left(E_{n}, \psi_{n}\right)$, the superpotential is given in terms of the ground state wave function $\psi_{0}$ by the relation (in units $\hbar=2 m=1$ )

$$
W(x)=-\frac{d}{d x}\left(\ln \psi_{0}\right),
$$

$W(x)$ being a solution of the nonlinear Riccati equation

$$
W^{2}-W^{\prime}(x)=V(x)-E_{0},
$$

obtained by inserting (2) into the Schrödinger equation $\psi_{n}^{\prime \prime}(x)+\left(E_{n}-V(x)\right) \psi_{n}(x)=0$ for $n=0$. Looking for a polynomial solution $W(y)$ of (3) expressed in the $y$ variable, we obtain [13]: $W(y)=$ $a y+b(a>0)$, where the coefficients $a$ and $b$ and the ground state energy $E_{0}$ are given by

$$
\begin{aligned}
a & = \pm \frac{\alpha}{2}+\sqrt{(\alpha / 2)^{2}+\lambda_{2}}, b=\frac{\lambda_{1}}{2 a}, \\
E_{0} & =\lambda_{0}+\alpha a-b^{2} .
\end{aligned}
$$

From the first equation, we get a relation which will be useful later

$$
a(a \mp \alpha)=\lambda_{2} .
$$

\subsection{Second category (generic)}

We say that a one-dimensional potential is a generic potential of second category [13] if the two following conditions are satisfied

$$
\left\{\begin{array}{l}
V(x) \rightarrow V(y)=\lambda_{2} y^{2}+\frac{\mu_{2}}{y^{2}}+\lambda_{0}, \quad \lambda_{2}, \mu_{2}>0 \\
y^{\prime}(x)=\alpha\left(1 \pm y^{2}\right)>0
\end{array}\right.
$$


This category contains the isotonic, Pöschl-Teller, Pöschl-Teller I, Pöschl-Teller II and Scarf potentials. Looking for an associated superpotential $W(y)$ solution of the Riccati equation in the $y$ variable, which is a Laurent polynomial, we obtain [13]: $W(y)=a y-\frac{b}{y}(a>0, b>0)$ where the unknown coefficients $a$ and $b$ and the ground state energy $E_{0}$ are then obtained as

$$
\begin{aligned}
a & = \pm \frac{\alpha}{2}+\sqrt{(\alpha / 2)^{2}+\lambda_{2}}, b=\frac{\alpha}{2}+\sqrt{(\alpha / 2)^{2}+\mu_{2}}, \\
E_{0} & =\lambda_{0}+2 a b+\alpha(a \pm b) .
\end{aligned}
$$

From (8), we get the two useful relations

$$
a(a \mp \alpha)=\lambda_{2}, b(b-\alpha)=\mu_{2} .
$$

\section{The quantization rules}

\subsection{The SWKB condition}

The SWKB quantization condition [1] is the supersymmetric version of the well known JWKB condition and allows to estimate the energy levels of $V(x)$ via the identity

$$
I^{S W K B}=\int_{x_{L}}^{x_{R}} \sqrt{E_{n}^{(-)}-W^{2}(x)} d x=n \pi
$$

where $x_{S L}$ and $x_{S R}$ are the SWKB turning points determined by $E_{n}^{(-)}=W^{2}(x)$ and $E_{n}^{(-)}$is related to the particle energy by $E_{n}^{(-)}=E_{n}-E_{0}$. Interestingly, the SWKB reproduces the exact energy spectrum for the complete set of PTSIP [11, which contains all the classical exactly solvable quantum potentials in closed analytical form.

In the following, we note $y_{S R}$ and $y_{S R}$, the $y$ turning points corresponding to the SWKB condition. After straightforward calculations, it results that they are determined, for the first category, by

$$
y_{S R}+y_{S L}=-\frac{E_{n}^{(-)}-b^{2}}{a^{2}}, \quad y_{S R} \cdot y_{S L}=-\frac{2 b}{a},
$$

consequently, the integral in (111) can be rewritten as

$$
I^{S W K B}=\frac{a}{\alpha} \int_{y_{S L}}^{y_{S R}} \sqrt{\left(y_{S R}-y\right)\left(y-y_{S L}\right)} \frac{d y}{1 \pm y^{2}} .
$$

For the second category and with the help of a second change of variable $z=y^{2}$, the $z$ turning points are

$$
z_{S R}+z_{S L}=\frac{E_{n}^{(-)}+2 a b}{a^{2}}, z_{S R} \cdot z_{S L}=\frac{b^{2}}{a^{2}}
$$

and for the integral in (11), we obtain

$$
I^{S W K B}=\frac{a}{2 \alpha} \int_{z_{S L}}^{z_{S R}} \sqrt{\left(z_{S R}-z\right)\left(z-z_{S L}\right)} \frac{d z}{z(1 \pm z)},
$$




\subsection{The Ma-Xu or proper quantization formula}

A few years ago, a semi-classical quantization rule was proposed by $\mathrm{Ma}$ and $\mathrm{Xu}$ [1, 2]

$$
\int_{x_{L}}^{x_{R}} \sqrt{E_{n}-V(x)} d x=n \pi+\gamma
$$

This rule generalizes the JWKB condition by the addition of a term $\gamma$ which takes into account the higher order quantum corrections and which appears to be necessarily energy independent for all the PTSIP [5]. It then gives a new way to access to the exact energy spectrum for this exactly solvable potential. In practice, it is first necessary to determine the energy $E_{0}$ of the ground state by solving the Riccati equation (3), then proceed to the evaluation of the integral $I^{P Q}\left(E_{n}\right)$ and replace $n=0$ to obtain $I^{P Q}\left(E_{0}\right)$.

Later Qiang and Dong have proposed a slightly improved version of the Ma-Xu formula, they called the proper quantization rule, which is given under the following form

$$
I^{P Q}\left(E_{n}\right)=I^{P Q}\left(E_{0}\right)+n \pi,
$$

where $I^{P Q}\left(E_{n}\right)$ is the integral in the left hand side of equation (16).

Let us now determine the $y$ turning points corresponding to proper quantization formula. For the first category, one obtains

$$
y_{P R}+y_{P L}=-\frac{E_{n}-\lambda_{0}}{\lambda_{2}}, \quad y_{P R} \cdot y_{P L}=-\frac{\lambda_{1}}{\lambda_{2}},
$$

and the integral (16) can be rewritten as

$$
I^{P Q}\left(E_{n}\right)=\frac{\sqrt{\lambda_{2}}}{\alpha} \int_{y_{P L}}^{y_{P R}} \sqrt{\left(y_{P R}-y\right)\left(y-y_{P L}\right)} \frac{d y}{1 \pm y^{2}} .
$$

For the second category, again with the help of a second change of variable $z=y^{2}$, the $z$ turning points are given by

$$
z_{P R}+z_{P L}=\frac{E_{n}-\lambda_{0}}{\lambda_{2}}, z_{P R} . z_{P L}=\frac{\mu_{2}}{\lambda_{2}}
$$

and the integral in (16) becomes

$$
I^{P Q}\left(E_{n}\right)=\frac{\sqrt{\lambda_{2}}}{2 \alpha} \int_{z_{P L}}^{z_{P R}} \sqrt{\left(z_{P R}-z\right)\left(z-z_{P L}\right)} \frac{d z}{z(1 \pm z)} .
$$

\section{From SWKB to proper quantization rule}

\subsection{First category}

We shall now investigate the relationship between $I^{S W K B}\left(E_{n}\right)$ and $I^{P Q}\left(E_{n}\right)$ for the first category of potentials. The presence of the symbol \pm in (13) means that we have in fact to evaluate two distinct integrals.

Starting from (13) and making use of the integral formulas (88) and (89) combined with expressions of the turning points given by (12), we obtain 


$$
I^{S W K B}= \begin{cases}\frac{\pi}{\sqrt{2} \alpha}\left(\sqrt{\left(E_{n}^{-}+a^{2}-b^{2}\right)^{2}+4 a^{2} b^{2}}+E_{n}^{-}+a^{2}-b^{2}\right)^{\frac{1}{2}}-\frac{\pi a}{\alpha}, & \text { if } y^{\prime}=\alpha\left(1+y^{2}\right), \\ \frac{\pi}{2 \alpha}\left[2 a-\sqrt{(a+b)^{2}-E_{n}^{-}}-\sqrt{(a-b)^{2}-E_{n}^{-}}\right] & , \text {if } y^{\prime}=\alpha\left(1-y^{2}\right) .\end{cases}
$$

In order to involve $I^{P Q}\left(E_{n}\right)$, we need to express the previous integrals in terms of $\lambda_{1}, \lambda_{2}$ and $E_{n}$.

In the first case, i.e. when $y^{\prime}(x)=\alpha\left(1+y^{2}\right)$, using equations (44) to (6) and $E_{n}^{(-)}=E_{n}-E_{0}$, it is straightforward to show that

$$
\left(E_{n}^{-}+a^{2}-b^{2}\right)+{ }^{2} 4 a^{2} b^{2}=\left(E_{n}-\lambda_{0}+\lambda_{2}\right)^{2}+\lambda_{1}^{2} .
$$

In the second case, i.e. when $y^{\prime}(x)=\alpha\left(1-y^{2}\right)$, we obtain in the same way

$$
(a \pm b)^{2}-E_{n}^{-}=\lambda_{2}+\lambda_{0}-E_{n} \mp \lambda_{1} .
$$

Inserting the two previous equations into (22), and after a little algebra, one finds

$$
I^{S W K B}=\left\{\begin{array}{l}
\frac{\pi \sqrt{\lambda_{2}}}{\alpha}\left[\frac{1}{\sqrt{2}}\left(\sqrt{\left(\frac{E_{n}-\lambda_{0}}{\lambda_{2}}+1\right)^{2}+\left(\frac{\lambda_{1}}{\lambda_{2}}\right)^{2}}+\frac{E_{n}-\lambda_{0}}{\lambda_{2}}+1\right)^{\frac{1}{2}}-1\right]-\frac{\pi}{\alpha}\left(a-\sqrt{\lambda_{2}}\right) \\
\frac{\pi \sqrt{\lambda_{2}}}{2 \alpha}\left[2-\sqrt{1+\frac{\lambda_{1}}{\lambda_{2}}-\frac{E_{n}-\lambda_{0}}{\lambda_{2}}}-\sqrt{1-\frac{\lambda_{1}}{\lambda_{2}}-\frac{E_{n}-\lambda_{0}}{\lambda_{2}}}\right]+\frac{\pi}{\alpha}\left(a-\sqrt{\lambda_{2}}\right) .
\end{array}\right.
$$

Using relations (19) and formulas (88) and (89), one can rewrite (25) as

$$
I^{S W K B}=\frac{\sqrt{\lambda_{2}}}{\alpha} \int_{y_{P L}}^{y_{P R}} \sqrt{\left(y_{P R}-y\right)\left(y-y_{P L}\right)} \frac{d y}{1 \pm y^{2}}-\left( \pm \frac{\pi}{\alpha}\left(a-\sqrt{\lambda_{2}}\right)\right) .
$$

Then, taking into account the fact that (see Appendix 1 for details)

$$
I^{P Q}\left(E_{0}\right)= \pm \frac{\pi}{\alpha}\left(a-\sqrt{\lambda_{2}}\right)
$$

and using (19), equation (26) becomes

$$
I^{S W K B}=I^{P Q}\left(E_{n}\right)-I^{P Q}\left(E_{0}\right),
$$

which means that the SWKB condition is identical to the proper quantization rule for the first category of PTSIP.

\subsection{Second category}

Starting from (15) and with the help of the integral formula (90) combined to expressions of the turning points given by (14), $I^{S W K B}$ can be written as

$$
I^{S W K B}=\frac{\pi}{2 \alpha}\left(\mp a-b \pm \sqrt{(b \pm a)^{2} \pm E_{n}^{-}}\right) .
$$


In order to make appear explicitly $I^{P Q}\left(E_{n}\right)$ in this expression, we can use the following identity (see (8) to (10))

$$
(b \pm a)^{2} \pm E_{n}^{-}= \pm E_{n} \mp \lambda_{0}+\lambda_{2}+\mu_{2} .
$$

Inserting (30) into (29) leads to

$$
I^{S W K B}=\frac{\sqrt{\lambda_{2}}}{2 \alpha} \pi\left[\left(\mp 1-\sqrt{\frac{\mu_{2}}{\lambda_{2}}} \pm \sqrt{1+\frac{\mu_{2}}{\lambda_{2}} \pm \frac{E_{n}-\lambda_{0}}{\lambda_{2}}}\right)-\left(\frac{b \pm a}{\sqrt{\lambda_{2}}} \mp 1-\sqrt{\frac{\mu_{2}}{\lambda_{2}}}\right)\right] .
$$

Refering to (20) and to the integral formulas (90), one finds

$$
I^{S W K B}=\frac{\sqrt{\lambda_{2}}}{2 \alpha} \int_{z_{P L}}^{z_{P R}} \sqrt{\left(z_{P R}-z\right)\left(z-z_{P L}\right)} \frac{d z}{z(1 \pm z)}-\frac{\sqrt{\lambda_{2}}}{2 \alpha}\left(\frac{b \pm a}{\sqrt{\lambda_{2}}} \mp 1-\sqrt{\frac{\mu_{2}}{\lambda_{2}}}\right) .
$$

But we have (see Appendix 1)

$$
I^{P Q}\left(E_{0}\right)=\frac{\pi}{2 \alpha}\left(b \pm a \mp \sqrt{\lambda_{2}}-\sqrt{\mu_{2}}\right),
$$

which leads to the expected result

$$
I^{S W K B}=I^{P Q}\left(E_{n}\right)-I^{P Q}\left(E_{0}\right),
$$

and proves that the two quantization rules are also equivalent for the second category PTSIP.

\section{Exceptional potentials}

In order to complete the study of the equivalence between the two quantization conditions, we still have to examine the four exceptional PTSIP [13] which necessitate a specific treatment. In the first category, the exceptional PTSIP are the harmonic potential, the Morse potential and Kepler-Coulomb potential. The isotonic potential is the unique exceptional potential belonging to the second category. In this section, the ground state energy $E_{0}$ is taken equal to zero.

\subsection{One-dimensional harmonic oscillator}

The one-dimensional harmonic potential with zero-energy ground state is [9], 15]

$$
V(x)=\frac{\omega^{2}}{4} x^{2}-\frac{\omega}{2}
$$

and the corresponding superpotential is [13] : $W(x)=\omega x / 2$. The SWKB turning points are $x_{S R}=-x_{S L}=(2 / \omega) \sqrt{E_{n}^{(-)}}$and those corresponding to the proper quantization are $x_{P R}=-x_{P L}=$ $(2 / \omega) \sqrt{E_{n}+\omega / 2}$. The evaluation of the $I^{S W K B}$ gives

$$
I^{S W K B}=\frac{\omega}{2} \int_{x_{S L}}^{x_{S R}} \sqrt{\left(x_{S R}-x\right)\left(x-x_{S L}\right)} d x=\frac{\omega}{2}\left(\frac{\pi}{8}\left(x_{S R}-x_{S L}\right)^{2}\right) .
$$

It is straightforward to prove that $\left(x_{S R}-x_{S L}\right)^{2}=\left(x_{P R}-x_{P L}\right)^{2}-\left(x_{P R 0}-x_{P L 0}\right)^{2}$ and since

$$
I^{P Q}\left(E_{n}\right)=\frac{\omega}{2} \int_{x_{P L}}^{x_{P R}} \sqrt{\left(x_{P R}-x\right)\left(x-x_{P L}\right)} d x
$$


we deduce immediately

$$
I^{S W K B}=I^{P Q}\left(E_{n}\right)-I^{P Q}\left(E_{0}\right)
$$

\subsection{Morse potential}

The Morse potential with zero-energy ground state is [9], [15]

$$
V(x)=A^{2}+B^{2} e^{-2 \alpha x}-2 B\left(A+\frac{\alpha}{2}\right) e^{-\alpha x},
$$

where the parameters $A, B$ and $\alpha$ are all positive. The introduction of the variable $y=e^{-\alpha x}$ transforms this potential into $V(y)=(A-B y)^{2}-\alpha B y$ and the corresponding superpotential is given by [13]: $W(y)=-B y+A$. The turning points corresponding to the SWKB are determined by

$$
\left\{\begin{array}{c}
y_{S R}+y_{S L}=2 A / B \\
y_{S R} \cdot y_{S L}=\left(A^{2}-E_{n}^{(-)}\right) / B^{2}
\end{array}\right.
$$

and those for the proper quantization by

$$
\left\{\begin{array}{l}
y_{P R}+y_{P L}=(2 A+\alpha) / B \\
y_{P R} \cdot y_{P L}=\left(A^{2}-E_{n}\right) / B^{2}
\end{array}\right.
$$

We have

$$
I^{S W K B}=\frac{B}{\alpha} \int_{y_{S L}}^{y_{S R}} \sqrt{\left(y_{S R}-y\right)\left(y-y_{S L}\right)} \frac{d y}{y}=\frac{B}{\alpha}\left[\frac{\pi}{2}\left(y_{S R}+y_{S L}\right)-\pi\left(y_{S R} \cdot y_{S L}\right)^{1 / 2}\right] .
$$

Combining this result with the preceding expressions for the turning points, we readily obtain

$$
I^{S W K B}=\frac{B}{\alpha}\left[\frac{\pi}{2}\left(y_{P R}+y_{P L}\right)-\pi\left(y_{P R} \cdot y_{P L}\right)^{1 / 2}-\frac{\pi}{2}\right] .
$$

Using

$$
\frac{\pi}{2}\left(y_{P R 0}+y_{P L 0}\right)-\pi\left(y_{P R 0} \cdot y_{P L 0}\right)^{1 / 2}=(\alpha / B) \frac{\pi}{2},
$$

and the integral formula

$$
I^{P Q}=(B / \alpha) \int_{y_{S L}}^{y_{S R}} \sqrt{\left(y_{P R}-y\right)\left(y-y_{P L}\right)} \frac{d y}{y}
$$

we then obtain

$$
I^{S W K B}=I^{P Q}\left(E_{n}\right)-I^{P Q}\left(E_{0}\right)
$$

\subsection{Kepler-Coulomb potential}

The Kepler-Coulomb potential with zero-energy ground state is [9], 15]

$$
V(x)=-\frac{\gamma}{x}+\frac{l(l+1)}{x^{2}}+\frac{\gamma^{2}}{4(l+1)^{2}} .
$$

Introducing the variable $y=1 / x$, it becomes $V(y)=-\gamma y+l(l+1) y^{2}+\gamma^{2} / 4(l+1)^{2}$ and the corresponding superpotential is given by [13] $W(y)=-a / x+b$, where $a=l+1>0$ and $b=$ $\gamma / 4(l+1)>0$. 
The SWKB turning points in terms of the $x$ variable satisfy

$$
\left\{\begin{array}{c}
x_{S R}+x_{S L}=2 a b /\left(b^{2}-E_{n}^{(-)}\right) \\
x_{S R} \cdot x_{S L}=a^{2} /\left(b^{2}-E_{n}^{(-)}\right)
\end{array}\right.
$$

and those for the proper quantization

$$
\left\{\begin{array}{c}
x_{P R}+x_{P L}=2 a b /\left(b^{2}-E_{n}\right) \\
x_{P R} \cdot x_{P L}=a(a-1) /\left(b^{2}-E_{n}\right) .
\end{array}\right.
$$

The integral for the SWKB formula in term of the $x$ variable is written as

$$
I^{S W K B}=\sqrt{b^{2}-E_{n}^{(-)}} \int_{y_{S L}}^{y_{S R}} \sqrt{\left(x_{S R}-x\right)\left(x-x_{S L}\right)} \frac{d x}{x} .
$$

Using the above expression of the different turning points, we show easily that

$$
\frac{\pi}{2}\left(x_{S R}+x_{P L}\right)-\pi\left(x_{S R} \cdot x_{S L}\right)^{1 / 2}=\frac{\pi}{2}\left(x_{P R}+x_{P L}\right)-\pi\left(x_{P R} \cdot x_{P L}\right)^{1 / 2}-\pi a / b+\pi a(a-1) / b,
$$

and

$$
\frac{\pi}{2}\left(x_{P R 0}+x_{P L 0}\right)-\pi\left(x_{P R 0} \cdot x_{P L 0}\right)^{1 / 2}=\pi a / b-\pi a(a-1) / b .
$$

Since we have also

$$
I^{P Q}=\sqrt{b^{2}-E_{n}} \int_{y_{S L}}^{y_{S R}} \sqrt{\left(x_{S R}-x\right)\left(x-x_{S L}\right)} \frac{d x}{x},
$$

we directly deduce

$$
I^{S W K B}=I^{P Q}\left(E_{n}\right)-I^{P Q}\left(E_{0}\right) .
$$

\subsection{Isotonic potential}

The isotonic potentiel with zero-energy ground state is [9], 15]

$$
V(x)=\frac{\omega^{2}}{4} x^{2}+\frac{l(l+1)}{x^{2}}-\omega\left(l+\frac{3}{2}\right)
$$

has an associated superpotential of the form [13] $W(x)=a x-b / x$, where $a=\omega / 2>0$ and $b=$ $l+1>0$.

The SWKB turning points in terms of $y=x^{2}$ are given by

$$
\left\{\begin{array}{c}
y_{S R}+y_{S L}=\left(2 a b+E_{n}^{(-)}\right) / a^{2} \\
y_{S R} \cdot y_{S L}=b^{2} / a^{2}
\end{array}\right.
$$

and those for the proper quantization are given by :

$$
\left\{\begin{array}{c}
y_{P R}+y_{P L}=\left(E_{n}+\omega(l+3 / 2)\right) / a^{2} \\
y_{P R} \cdot y_{P L}=b(b-1) / a^{2}
\end{array}\right.
$$


In terms of the $y$ variable, the SWKB integral takes the form

$$
I^{S W K B}=\frac{a}{2} \int_{y_{S L}}^{y_{S R}} \sqrt{\left(y_{S R}-y\right)\left(y-y_{S L}\right)} \frac{d y}{y}=\pi \frac{E_{n}^{(-)}}{2 \omega} .
$$

Similarly, we have also

$$
I^{P Q}=\frac{a}{2} \int_{y_{S L}}^{y_{S R}} \sqrt{\left(y_{P R}-y\right)\left(y-y_{P L}\right)} \frac{d y}{y}=\pi\left(E_{n}^{(-)} / 2 \omega+\left(l+\frac{3}{2}\right) / 2\right),
$$

from which we can directly conclude that

$$
I^{S W K B}=I^{P Q}\left(E_{n}\right)-I^{P Q}\left(E_{n=0}\right),
$$

for the isotonic potential.

\section{$5 \quad$ Energy spectra of the PTSIP}

\subsection{First category PTSIP}

For the first category of potentials, the calculation of the energy spectrum is achieved by solving equation $I^{S W K B}=n \pi$, where $I^{S W K B}$ is given by (22). Consider the two equations in (22) and introduce the notations $\eta_{1}=E_{n}^{-}+a^{2}-b^{2}$ into the first equation and $\eta_{2}=\sqrt{(a+b)^{2}-E_{n}^{-}}$into the second one. The substitution of these variables into the identity $I^{S W K B}=n \pi$ gives correspondingly

$$
\left\{\begin{array}{c}
\sqrt{\eta_{1}^{2}+4 a^{2} b^{2}}+\eta_{1}=2(a+\alpha n)^{2} \\
-\sqrt{\eta_{2}^{2}-4 a^{2} b^{2}}-\eta_{2}=2(\alpha n-a)
\end{array} .\right.
$$

Since $\xi=2(a+\alpha n)^{2}$ is one of the two solutions of the quadratic equation $(1 / 2) \xi^{2}-\eta_{1} \xi-2 a^{2} b^{2}=0$, it is sufficient to solve the latter with respect to $\eta_{1}$. Following the same method to find $\eta_{2}$, one obtaines the analytical expression of energy levels

$$
E_{n}=\mp a^{2}+\alpha a+\lambda_{0} \pm(a \pm \alpha n)^{2}-\frac{\lambda_{1}^{2}}{4(a \pm \alpha n)^{2}} .
$$

Equation (6) allows us to express the energy as a function of the parameters $\lambda_{2}, \lambda_{1}, \lambda_{0}$ and $\alpha$. To illustrate this result, we consider the Rosen-Morse I and II potentials and the Eckart potential.

\subsubsection{Rosen-Morse I}

The Rosen-Morse I potential with zero-energy ground state has the form [9], [15]

$$
V(x)=\frac{A(A-\alpha)}{\sin ^{2}(\alpha x)}+2 B \cot (\alpha x)-A^{2}+\frac{B^{2}}{A^{2}}, 0 \leq \alpha x \leq \pi .
$$

Using the variable $y=-\cot (\alpha x)\left(y^{\prime}(x)=\alpha\left(1+y^{2}\right)\right)$, it becomes $V(y)=A(A-\alpha) y^{2}-2 B y+$ $B^{2} / A^{2}-\alpha A$. Comparing with (10) gives

$$
a=A, b=-\frac{B}{A}
$$


from which, we deduce for the superpotential $W(x)=a y+b=A(-\cot (\alpha x))-B / A$ and for the energy levels

$$
E_{n}=-A^{2}+\frac{B^{2}}{A^{2}}+(A+\alpha n)^{2}-\frac{B^{2}}{(A+\alpha n)^{2}}
$$

\subsubsection{Rosen-Morse II}

The Rosen-Morse II potential with zero-energy ground state is given by [9], 15]

$$
V(x)=-\frac{A(A+\alpha)}{\cosh ^{2}(\alpha x)}+2 B \tanh (\alpha x)+A^{2}+\frac{B^{2}}{A^{2}}, \quad B<A^{2} .
$$

Using the variable $y=\tanh (\alpha x)\left(y^{\prime}(x)=\alpha\left(1-y^{2}\right)\right)$, it becomes $V(y)=A(A+\alpha) y^{2}+2 B y+$ $B^{2} / A^{2}-\alpha A$. Comparing with (11) gives

$$
a=A, b=\frac{B}{A},
$$

which leads to a superpotential $W(x)=A \tanh (\alpha x)+B / A$ and the energy spectrum is

$$
E_{n}=A^{2}+\frac{B^{2}}{A^{2}}-(A-\alpha n)^{2}-\frac{B^{2}}{(A-\alpha n)^{2}}
$$

\subsubsection{Eckart potential}

The Eckart potential with zero-energy ground state is given by [9], [15]:

$$
V(x)=\frac{A(A-\alpha)}{\sinh ^{2}(\alpha x)}-2 B \operatorname{coth}(\alpha x)+A^{2}+\frac{B^{2}}{A^{2}}, B>A^{2} .
$$

Using the variable $y=\operatorname{coth}(-\alpha x)$ and redefining $\alpha \rightarrow-\alpha$, it becomes $V(y)=A(A+\alpha) y^{2}+$ $2 B y+B^{2} / A^{2}-\alpha A$ which is exactly the form obtained in the previous case. The superpotential is then $W(x)=A \operatorname{coth}(-\alpha x)+B / A$ and the energy levels are simply obtained by replacing $\alpha$ by $-\alpha$ in (66)

$$
E_{n}=A^{2}+\frac{B^{2}}{A^{2}}-(A+\alpha n)^{2}-\frac{B^{2}}{(A+\alpha n)^{2}} .
$$

\subsection{Second category PTSIP}

For the second category of potentials, the resolution of $I^{S W K B}=n \pi$ leads to the following expression for energy levels [24]

$$
E_{n}=\lambda_{0} \mp\left(\mu_{2}+\lambda_{2}\right) \pm(2 \alpha n \pm a+b)^{2}
$$

\subsubsection{Pöschl-Teller}

The Pöschl-Teller potential with zero-energy ground state is given by [9], 15]

$$
V(x)=A^{2}+\frac{A^{2}+B^{2}+\alpha A}{\sinh ^{2}(\alpha x)}-B(2 A+\alpha) \frac{\operatorname{coth}(\alpha x)}{\sinh (\alpha x)}, \quad B>A, \quad x>0 .
$$


The choice of variable $y=\tanh (\alpha x / 2)$ transforms it into $V(y)=a(a+\alpha / 2) y^{2}-b(b-\alpha / 2) / y^{2}+\lambda_{0}$, where

$$
a=\frac{A+B}{2}, \quad b=\frac{B-A}{2}
$$

and $\lambda_{0}=-\alpha(a-b) / 2-2 a b$. In this case $y^{\prime}(x)=\alpha\left(1-y^{2}\right) / 2$, and one obtains as superpotential $W(x)=a y-b / y=A \operatorname{coth}(\alpha x)-B / \sinh (\alpha x)$ and as energy spectrum (see (69))

$$
E_{n}=A^{2}-(\alpha n-A)^{2} .
$$

\subsubsection{Pöschl-Teller I}

The Pöschl-Teller I potential with zero-energy ground state has the form [9], [15]

$$
V(x)=-(A+B)^{2}+\frac{A(A-\alpha)}{\cos ^{2}(\alpha x)}+\frac{B(B-\alpha)}{\sin ^{2}(\alpha x)}, \quad 0<x<\frac{\pi}{2 \alpha}
$$

Introducing the variable $y=\tan \alpha x\left(y^{\prime}(x)=\alpha\left(1+y^{2}\right)\right)$, this potential takes the form $V(y)=$ $a(a-\alpha) y^{2}-b(b-\alpha) / y^{2}+\lambda_{0}$, where

$$
a=A, b=B
$$

and $\lambda_{0}=-\alpha(a+b)-2 a b$. One obtains $W(x)=a y-b / y=A \tan (\alpha x)-B \cot (\alpha x)$ and the energy spectrum is (see (69))

$$
E_{n}=-(A+B)^{2}+(2 \alpha n+A+B)^{2} .
$$

\subsubsection{Poschl-Teller II}

The Pöschl-Teller II potential with zero-energy ground state has the following form [9], [15]

$$
V(x)=(A-B)^{2}-\frac{A(A+\alpha)}{\cosh ^{2}(\alpha x)}+\frac{B(B-\alpha)}{\sinh ^{2}(\alpha x)}, B<A, x>0 .
$$

By using the variable $y=\tanh \alpha x$, we obtain $V(y)=a(a+\alpha) y^{2}+b(b-\alpha) / y^{2}+\lambda_{0}$, where

$$
a=A, b=B,
$$

and $\lambda_{0}=-\alpha(a-b)-2 a b$. The superpotential becomes $W(x)=A \tanh (\alpha x)-B \operatorname{coth}(\alpha x)$ and the energy levels are given by

$$
E_{n}=(B-A)^{2}-(2 \alpha n+B-A)^{2} .
$$

\subsubsection{Scarf I}

The Scarf I potential with zero-energy ground state is given by [9], [13]

$$
V(x)=-A^{2}+\frac{A^{2}+B^{2}-\alpha A}{\sin ^{2}(\alpha x)}-B(2 A-\alpha) \frac{\cot (\alpha x)}{\sin (\alpha x)}, B<A, 0<x<\frac{\pi}{2 \alpha},
$$

With the introduction of the variable $y=\tan (\alpha x / 2)$, it takes the form $V(y)=a\left(a-\frac{\alpha}{2}\right) y^{2}+b(b-$ $\alpha / 2) / y^{2}+\lambda_{0}$, where

$$
a=\frac{A+B}{2}, b=\frac{A-B}{2}
$$

and $\lambda_{0}=-\alpha(a+b) / 2-2 a b$. In this case $y^{\prime}(x)=\alpha\left(1+y^{2}\right) / 2$ and one obtains the superpotential $W(x)=-A / \tan (\alpha x)+B / \sin (\alpha x)$. The energy levels are deduced from (69) as

$$
E_{n}=-A^{2}+(A+\alpha n)^{2} \text {. }
$$




\section{Conclusion}

In this work, we have examined the link between the Ma-Xu or proper quantization rule and the SWKB condition. By using the classification into two categories of the PTSIP, we have shown the equivalence of these two quantization rules. The study covers the whole set of PTSIP, including the exceptional cases. As an application of the general classification introduced in [13], a global formula for the energy levels of every PTSIP has been extracted from the SWKB, recovering in each specific case the known results [10, 11].

A challenging problem for the future would be to establish exact quantization formulas for the solvable potentials obtained as rational extensions of the PTSIP considered above [6, 18, 19, 20, 21, 22. It seems indeed that the SWKB quantization formula is not exact for these potentials even for those which belong to the class of TSIP [12]. This has to be correlated to the $\hbar$ dependence of the superpotentials associated to these extensions in contrast with the primary TSIP for which the superpotential does not present such dependence $[23]$.

\section{Appendix 1 - Evaluation of $I^{P Q}\left(E_{0}\right)$}

\subsection{First category}

Taking $n=0$ in the first terms of the two equations of (25), one finds

$$
I^{P Q}\left(E_{0}\right)=\left\{\begin{array}{c}
\frac{1}{\alpha}\left[\frac{\pi}{\sqrt{2}}\left(\sqrt{\left(E_{0}-\lambda_{0}+\lambda_{2}\right)^{2}+\lambda_{1}^{2}}+E_{0}-\lambda_{0}+\lambda_{2}\right)^{\frac{1}{2}}-\sqrt{\lambda_{2}} \pi\right], \text { if } y^{\prime} / \alpha=1+y^{2}, \\
\frac{\pi}{2 \alpha}\left[2 \sqrt{\lambda_{2}}-\sqrt{\lambda_{2}+\lambda_{1}+\lambda_{0}-E_{0}}-\sqrt{\lambda_{2}-\lambda_{1}+\lambda_{0}-E_{0}}\right], \text { if } y^{\prime} / \alpha=1-y^{2} .
\end{array}\right.
$$

Using (44) to (6), it is straightforward show that

$$
I^{P Q}\left(E_{0}\right)= \begin{cases}\left(\sqrt{\left(E_{0}-\lambda_{0}+\lambda_{2}\right)^{2}+\lambda_{1}^{2}}+E_{0}-\lambda_{0}+\lambda_{2}\right)^{\frac{1}{2}}=\sqrt{2} a, & \text { if } y^{\prime}=\alpha\left(1+y^{2}\right), \\ \sqrt{\lambda_{2} \pm \lambda_{1}+\lambda_{0}-E_{0}}=|a \pm b|, & \text { if } y^{\prime}=\alpha\left(1-y^{2}\right),\end{cases}
$$

and inserting these identities into (82) we obtain

$$
I^{P Q}\left(E_{0}\right)=\left\{\begin{array}{cc}
\frac{\pi}{\alpha}\left(a-\sqrt{\lambda_{2}}\right), & \text { if } y^{\prime} / \alpha=1+y^{2}, \\
-\frac{\pi}{\alpha}\left(a-\sqrt{\lambda_{2}}\right),(b<a) & \text { if } y^{\prime} / \alpha=1-y^{2}
\end{array}\right.
$$

which is the desired result.

\subsection{Second category}

As we have seen, the first terms of the right-hand side of equation (31) correspond to $I^{P Q}\left(E_{n}\right)$. For $n=0$, the expression of $I^{P Q}\left(E_{0}\right)$ is written under the following form

$$
I^{P Q}\left(E_{0}\right)=\frac{\sqrt{\lambda_{2}}}{2 \alpha} \pi\left[\left(\mp 1-\sqrt{\frac{\mu_{2}}{\lambda_{2}}} \pm \sqrt{1+\frac{\mu_{2}}{\lambda_{2}} \pm \frac{E_{0}-\lambda_{0}}{\lambda_{2}}}\right)\right] .
$$


By means of (13) and taking $n=0$, one gets

$$
(b \pm a)^{2}= \pm E_{0} \mp \lambda_{0}+\lambda_{2}+\mu_{2} .
$$

Substituting the last relation in (23) leads to

$$
I^{P Q}\left(E_{0}\right)=\frac{\pi}{2 \alpha}\left(b \pm a \mp \sqrt{\lambda_{2}}-\sqrt{\mu_{2}}\right),
$$

where $b<a$ in the second case, i.e. when $y^{\prime}(x)=\alpha\left(1-y^{2}\right)$.

\section{Appendix 2}

For the first category of potentials, the following integral formulas [14 have been used

$$
\begin{aligned}
& \int_{\alpha}^{\beta} \sqrt{(\beta-y)(y-\alpha)} \frac{d y}{1+y^{2}}=\frac{\pi}{\sqrt{2}}\left[\sqrt{1+\alpha^{2}} \sqrt{1+\beta^{2}}-\alpha \beta+1\right]^{1 / 2}-\pi, \alpha<\beta, \\
& \int_{\alpha}^{\beta} \sqrt{(\beta-y)(y-\alpha)} \frac{d y}{1-y^{2}}=\frac{\pi}{2}[2-\sqrt{(1-\alpha)(1-\beta)}-\sqrt{(1+\alpha)(1+\beta)}],
\end{aligned}
$$

where $-1<\alpha<\beta<1$ for the second formula. For the second category of potentials, we have the following formula 24 ]

$$
\int_{\alpha}^{\beta} \sqrt{(\beta-z)(z-\alpha)} \frac{d z}{z(1 \pm z)}=\pi[\mp 1-\sqrt{\alpha \beta} \pm \sqrt{(1 \pm \alpha)(1 \pm \beta)}]
$$

The signs \pm in the last integral represents two different cases and the formula is valid under the following condition: the parameters $\alpha$ and $\beta$ are positive in both cases and are lower than unity in the second.

\section{References}

[1] Z. Q. Ma and B. W. Xu, Int. J. Mod. Phys. E 14, 599-610 (2005).

[2] Z. Q. Ma and B. W. Xu, Europhys. Lett. 69, 685-691 (2005).

[3] W. C. Qiang and S. H. Dong, EPL 89, 10003 (2010).

[4] Y. Kasri and L. Chetouani, Can.J. Phys. 86, 1-7 (2008).

[5] Y. Grandati and A. Bérard, Phys. Lett. A 375, 390-395 (2011).

[6] Y. Grandati, Ann. Phys. 326, 2074-2090 (2011) ; Y. Grandati, J. Phys. Conf. Ser. 343, 012041 (2012) ;

[7] Y. Grandati, Ann. Phys. 327, 2411-2431 (2012).

[8] Y. Grandati and A. Bérard, J. Eng. Math. 82, 161-171 (2013) ;

[9] F. Cooper, A. Khare and U. Sukhatme, Supersymmetry in Quantum Mechanics, (World Scientific, Singapore, 2001) 
[10] A. Comtet, A. Bandrauk, and D. K. Campbell, Phys. Lett. B 150, 159 (1985) ; A. Khare, ibid. 161, 131 (1985).

[11] R. Dutt, A. Khare and U. Sukhatme, Phys. Lett. B 181, 295 (1986) ; R. Dutt, A. Khare and U. P. Sukhatme, Am. J. Phys. 59, 723-727 (1991).

[12] R. S. Bhalla, A. K. Kapoor and P. K. Panigrahi, Am J. Phys. 65, 1187 (1997) ; C. Cherqui, Y. Binder and A. Gangopadhyaya, Phys. Lett. A 372, 1406 (2008) ; S. Sree Ranjani, P. K. Panigrahi, A. Khare, A. K. Kapoor and A. Gangopadhyaya, J. Phys. A 45, 055210 (2012).

[13] Y. Grandati and A. Bérard, Ann. Phys. 325, 1235-1259 (2010).

[14] M. Hruška, W.Y. Keung and U. Sukhatme. Phys. Rev. A 55, 5 (1997).

[15] R. Dutt, A. Khare and U.P. Sukhatme, Am. J. Phys. 56, 163-168 (1988).

[16] L. Gendenshtein, JETP Lett. 38, 356-359 (1983).

[17] D. T. Barclay, Phys Lett. A 185, 169-173 (1994).

[18] Y. Grandati, J. Math. Phys. 52, 103505 (2011) ; Y. Grandati, Phys. Lett. A 376, 2866-2872 (2012).

[19] C. Quesne, J. Phys. A Math. Theor. 41, 392001 (2008) ; C. Quesne, SIGMA 5, 084 (2009) ; C. Quesne, Mod. Phys.Lett. A 26 1843-1852 (2011) ; C. Quesne, Int. J. Mod. Phys. A 27, 1250073 (2012). ; C. Quesne, SIGMA 8080 (2012); Y. Grandati and C. Quesne, J. Math. Phys. 54, 073512 (2013).

[20] D. Gomez-Ullate, N. Kamran and R. Milson, J. Phys. A 37, 1789-1804 (2004) ; D.Gomez-Ullate, N. Kamran and R. Milson, J. Phys. A 43, 434016 (2010) ; D. Gomez-Ullate, Y. Grandati and R. Milson, J. Phys. A: Math. Theor. 47 015203, (2014).

[21] A. Ramos, J. Phys. A 44, 342001 (2011) ; A. Ramos, Phys. Lett. A 376, 3499-3503 (2012).

[22] S. Odake and R. Sasaki,, Phys. Lett. B 679, 414-417 (2009) ; S. Odake and R. Sasaki, Phys. Lett. B 684, 173-176 (2009) ; S. Odake and R. Sasaki, Phys. Lett. B 702 164-170 (2011).

[23] J. Bougie, A. Gangopadhyaya and J. V. Mallow, Phys. Rev. Lett. 105, 210402 (2010) ; J. Bougie, A. Gangopadhyaya and J. V. Mallow, J. Phys. A: Math. and Theor. 44, 275307 (2011) .

[24] N. Benchiheub,Y. Kasri, A. Kahoul, Canadian Journal of Physics, 93(12), 1486-1489 (2015) 